\title{
ELECTRICITY AND RADIATIONS IN REHABILITATION FROM WAR INJURIES
}

\author{
By P. BAUWENS \\ (Medical Officer in charge Electro-therap. Dept., St. Thomas's Hospital; Hon. Electro-therap., Royal \\ Westminster Ophthalmic Hospital)
}

In its broadest sense, rehabilitation embraces any step taken with a view to restoring the injured or the sick to the nearest approach to fitness. Such steps may include surgical interference, psychological measures, and the use of physical methods either singly or in combination.

When indicated, the application of electrical and radiant energy for rehabilitation purposes generally precedes the use of the more active physical methods such as remedial exercises, games and occupations which are more directly concerned with restoration of activity by encouraging function. The value of electro- and photo-therapeutic methods spring from the fact that some of the conditions which retard repair and hinder recovery of function are amenable to the action of physical agents.

The conditions which retard repair are: sepsis; absence of stimuli required for the initiation and maintenance of repair processes; deficient local circulation; low temperature and poor general condition of the patient. Those which interfere with recovery of function are: pain, persistent exudate leading to organisation, adhesions and unbalanced muscle action.

When used in a rational manner, physical agents can be of the greatest help in combating these conditions by altering the chemical structure of protoplasm, by raising the temperature of tissues, by modifying the ionic content of intercellular fluids, or by producing movement.

Sepsis in accessible situations can be dealt with by direct sterilisation by means of ultraviolet rays. For this purpose the short radiations from 3,000-2,500 Angström units are employed. As these are not penetrating, the bactericidal effect is entirely superficial. A deeper sterilisation can be achieved by driving zinc ions into the infected tissues under the positive electrode. With this procedure, the zinc ions, when in the intercellular spaces, regain their atomic character and combine with the proteins of the intercellular fluids to form a zinc proteinate coagulum which constitutes a medium unsuitable for the development of pathogenic organisms.

Both these methods of treatment exert a secondary action by producing inflammatory reactions in the neighbouring tissues. The resulting stimulation of cellular activity increases the defensive processes.

Where sepsis is the sole cause of indolence, its removal may bring about subsequent healing, otherwise this can be accelerated by further irradiation with ultra-violet rays. Here again it is the lethal properties of these radiations which are being exploited. I.ittle doubt exists now that the stimulus responsible for bringing about proliferation of cells is provided by substances originating in injured cells. This is in accordance with the work of Carel and others. It has lately been confirmed by Nutini that the accelerated repair consequent upon treatment with ultra-violet rays is the indirect result of the lethal action of these radiations on the superficial tissues.

Neither defensive nor healing processes can take place in the presence of inadequate circulation or lowered temperature. These two conditions generally co-exist and can often be combated by the same agent; for increase in temperature is one of the best vaso-dilator stimuli. while vaso-dilatation, when thorough, will cause a local rise in temperature. A combined rise in temperature and increased blood supply may be brought about by warming up large areas of skin. Infra-red rays and luminous rays are often employed for this purpose. These thermogenic radiations, when absorbed by the superficial tissues, are converted into heat, and not only cause a rise in temperature superficially but cause a deeper reflex vaso-dilatation. Where nerve lesions exist and degeneration of the afferent nerve fibres has occurred, axon reflexes and other reflex vaso-motor reactions are not elicited.

Deep heating, with consequent vaso-dilatation of large vessels, can be achieved by means of high-frequency currents. This is best accomplished in the short wave field provided by a cable coiled around the limb in a spiral fashion. Advantages accrue from this method on account of the absence of steep temperature gradients and from the fact that it may be used 
even though the limb be encased in plaster, provided the splint does not embody any metal and is not sodden.

A rise in temperature will cause an acceleration of chemical activity. Within the limits of tissue stability, it will accelerate cellular activity and biochemical reactions. Defensive processes are a combination of both, and may therefore be expected to be promoted very considerably by an increase in temperature. It must, however, be remembered that a rise in temperature does also increase the activity of pathogenic organisms. Again, an increase in temperature in a limb inadequately supplied with blood may precipitate gangrene, by increasing the demand for oxygen and nutritive material to an extent in excess of supply and by at the same time promoting pathological processes.

Repair processes may be delayed by a poor general condition due to deficient calcium metabolism or by a defective response of the anti-body producing tissues. Once more ultraviolet rays may be of use on account of their property of converting pro-vitamin $D$ into vitamin $\mathrm{D}$ and of provoking esophylactic reactions. This term is applied to the increased anti-body production in skin which is in a state of mild inflammation (first degree erythema) caused by previous irradiation with ultra-violet rays.

Pain, whether real, exaggerated or imaginary, may constitute one of the greatest barriers to restoration of function during repair, or even after this is completed. One of the most intractable conditions of this order is causalgia. According to Turrell of Oxford, the electrotherapeutic method which gives the greatest relief from this distressing symptom is the Leduc current-a unidirectional intermittent current of low intensity.

Reflex neuralgic pains are frequently relieved by driving in calcium ions under the positive electrode. It is probable that this ion raises the threshold of excitability and irritability.

Even trivial injuries may. have grave consequences if oedema and extravasation of blood persist and lead to organisation. For this reason they should be dealt with at the earliest possible moment. Movement, unless contra-indicated for other reasons, is one of the best methods of assisting resorption of exudates. It is obtained by inducing graduated muscular contractions with the faradic current. These contractions should alternate with periods of rest at the rate of about 20 per minute at first, progressively increasing to 30 or 40 per minute. The depleting effect of this treatment may be enhanced by exerting pressure on the affected part either by firm bandaging or the application of sandbags during the treatment.

The application of galvanism to the elevated limb, preferably with the positive electrode over the swollen portion, will help to reduce the swelling, and may usefully be combined with the foregoing methods.

Adhesions when superficial can be softened and loosened from neighbouring structures by driving chlorine or iodine ions into them. This is done by means of the galvanic current, under the negative electrode, and should be supplemented by oral intake of iodine.

Deeper matting of tissues and induration should be treated by means of X-rays or radium, both of which have a potent fibrolytic action.

Where structures concerned with movement are involved, it is important to combine the preceding treatments with exercises, whether artificially induced or natural.

Sensory nerve twigs are occasionally involved in contracting scar tissue or form small neuromata after injury. The highly sensitive spots thus produced, if subcutaneous, can be destroyed by galvanic needling. The rationale of this treatment is based on the destruction of the fibrous tissue or the neuroma by the sodium hydroxide liberated around the cathode when a galvanic current is applied to tissues with bare electrodes. After the sensitive spot has been accurately localised and anaesthetised, it is transfixed with a steel needle connected to the negative pole of a galvanic battery and a current of 3-5 ma. passed.

Injuries to nerves, muscles, tendons, bones and joints are often accompanied by, or result in, unbalanced muscle action. This may be brought about by complete or partial paralysis due to nerve injury, spasm of one or more muscle groups, reflex or disuse atrophy or vicious use of some muscles.

It is well known that on account of reciprocal innervation, atrophy of one muscle, whether due to nerve injury or disuse atrophy, produces increased tone in its antagonist. This phenomenon can be observed every.day in the thigh muscles, where wasting of the extensors of the knee rapidly results in a hypertonic state in the flexors. The increased tone in the hamstrings leads to further inhibition of the quadriceps, and thus the vicious circle is maintained. When this state of affairs has reached a certain threshold it is wellnigh impossible to restore the 
balance by the sole use of exercises, possibly because the weakened muscles are incapable of producing the afferent stimuli necessary to bring about inhibition of the hypertonic antagonists. Graduated muscular contractions induced by means of faradic currents not only exercise the muscle but stimulate the sensory end organs in the muscle, thus artificially supplementing the deficient afferent impulses.

Where nerve injury with axon degeneration accounts for the atony and wasting, the muscles will not respond to faradic stimulation and interrupted galvanic current has to be resorted to.

In some cases re-education of muscle sense and sense of movement is necessary, as, for instance, after tendon transplantation. This may be facilitated in the earlier stages by utilising faradic stimulation of the muscles involved.

As suggested at the beginning of this article, electro-therapy constitutes but a small aspect of rehabilitation, but it is an important one. Many of the methods empioyed are passive ones from the patient's point of view, and therefore they should as soon as expedient be replaced by or combined with more active treatments which should eventually supplant them.

\title{
THE REHABILITATION OF NON-TUBERCULOUS DISEASE AND INJURY OF THE CHEST
}

\author{
By F. S. COOKSEY, M.D.(Lond.) \\ (Medical Officer in charge of rehabilitation, Horton Hospital, E.M.S.)
}

The word rehabilitation has come to be so widely used and diversely applied that it is necessary to clarify the use of the term. Taken in a broad sense it is a convenient omnibus term for a number of positive measures directed towards the restoration of health and social normality. These measures may be enumerated as (I) medical and surgical practice to promote recovery from the effects of disease or injury; (2) ancillary therapeutic methods to assist restoration of function in the disabled part together with the restoration of normal physique in the body as a whole; (3) social organisation to provide freedom from want during illness, adequate domicilary or hospital treatment with eventual return to normal employment; (4) provision for the vocational training of the permanently disabled followed by guaranteed employment, if necessary in sheltered industries.

There is nothing new in the conception or practice of rehabilitation. Indeed the principles are the foundation of good doctoring and as old as the art itself whilst, in the thoracic field, the pioneer work of Papworth and Preston Hall have set a standard exceeded in no other branch. What is new, perhaps, is the effort to co-ordinate the medical and sociological aspects and to apply the principles and practice to the field of general medicine and surgery. The present article is concerned mainly with the application of the ancillary therapeutic methods in the rehabilitation of disease and injury of the chest; but excluding tuberculosis which presents quite separate and highly specialised problems. The ancillary therapeutic methods referred to are physiotherapy, occupational therapy, group exercises and remedial games. Brief reference will be made also to the use of mental exercise or educational therapy.

\section{Breathing exercises.}

Recovery from thoracic illness is dependent, in the first instance, upon the restoration of respiratory function hence it is not surprising that breathing exercises are the predominant therapy.

(a) Inspiratory or lung expansion exercises are used in atelectasis, pneumonia, empyema, after lob- and pneumon-ectomy and as a prophylactic before and after operations on the upper abdomen, etc. Two methods are available; firstly, forced expiration against resistance such as the closed glottis or Wolff's bottles, and secondly, inspiratory breathing exercises. In the former it is difficult to avoid the effort being expended on over-expansion of the normal lung or even the production of emphysema, since the positive pressure is distributed to all parts of the lung and the diseased or damaged area usually offers greater resistance to expansion due to organised fibrinous exudate. In the latter use is made of the negative pressure created by the elevation of the ribs and descent of the diaphragm in normal respiration and the effort is local- 